\title{
Analysis of $\mathrm{Gd}(\mathrm{n}$,gamma) reaction with 155,157 and natural Gd targets taken with JPARC-ANNRI and development of $\mathrm{Gd}(\mathrm{n}, \mathrm{gamma})$ decay model for Gd-doped neutron/neutrino detectors
}

\author{
Ali Ajmi ${ }^{* 1,2}$, T. Tanaka ${ }^{1}$, K. Hagiwara ${ }^{1}$, T. Sudo ${ }^{1}$, M. Reen ${ }^{1}$, P. Das ${ }^{1}$, R. Dir ${ }^{1}$, \\ Y. Yamada ${ }^{1}$, I. Ou ${ }^{1}$, T. Mori ${ }^{1}$, T. Kayano ${ }^{1}$, Y. Koshio ${ }^{1}$, M. Sakuda ${ }^{1}$, T. Yano ${ }^{3}$, \\ A. Kimura ${ }^{4}$, S. Nakamura ${ }^{4}$, N. Iwamoto ${ }^{4}$, H. Harada ${ }^{4}$, S. Lorenz ${ }^{5}$, M. Wurm ${ }^{5}$, \\ W. Focillon ${ }^{6}$, M. Gonin ${ }^{6}$, G. Collazuol ${ }^{2}$ \\ ${ }^{1}$ Okayama University, ${ }^{2}$ University of Padova and INFN, ${ }^{3}$ Kobe University, ${ }^{4}$ Japan Atomic \\ Energy Agency, ${ }^{5}$ Johannes Gutenberg-Universität Mainz, ${ }^{6}$ École Polytechnique \\ E-mail: aliajmi@okayama-u.ac.jp
}

\begin{abstract}
The importance of a good model for the $\gamma$-ray energy spectrum from the radiative thermal neutron capture on Gadolinium (Gd) is specially increased in the present era of Gd-enhanced $\bar{v}_{e}$-search detectors. Its an essential prerequisite for MC studies to evaluate the neutron tagging efficiency, in order to enhance signal sensitivity in the Gd-loaded $\bar{v}_{e}$-search detectors. The $\gamma$-ray spectra produced from the thermal neutron capture on enriched gadolinium targets $\left({ }^{155} \mathrm{Gd},{ }^{157} \mathrm{Gd}\right.$ and Natural Gd) in the energy range $0.11 \mathrm{MeV}$ to $8.0 \mathrm{MeV}$, were measured using the ANNRI Germanium Spectrometer at MLF, J-PARC. Based on the data acquired and a GEANT4 simulation of the ANNRI detector, we reported the energy spectrum of ${ }^{157} \mathrm{Gd}(\mathrm{n}, \gamma)$ and developed a $\gamma$-ray emission model of ${ }^{157} \mathrm{Gd}(\mathrm{n}, \gamma)$ in our previous publication. We now present the analysed data of ${ }^{155} \mathrm{Gd}(\mathrm{n}$, $\gamma)$ and ${ }^{\text {nat }} \mathrm{Gd}(\mathrm{n}, \gamma)$ reactions, the energy spectra of $\gamma$-rays and an improved model for ${ }^{155} \mathrm{Gd}(\mathrm{n}, \gamma)$, ${ }^{157} \mathrm{Gd}(\mathrm{n}, \gamma)$ and ${ }^{\text {nat }} \mathrm{Gd}(\mathrm{n}, \gamma)$ reactions. The consistency of the results from the devised model is checked among all the 14 germanium crystals, at the level of $15 \%$ spectral shape deviation at 0.2 $\mathrm{MeV}$ binning.
\end{abstract}

The 39th International Conference on High Energy Physics (ICHEP2018)

4-11 July, 2018

Seoul, Korea

${ }^{*}$ Speaker. 


\section{Introduction}

Gadolinium (Gd) is being used in a number of neutrino experiments for enhanced detection of electron anti-neutrinos $\left(\bar{v}_{e}\right)$ through neutron-tagging in the inverse beta decay reaction $\left(\bar{v}_{e}+p \rightarrow\right.$ $\left.e^{+} n\right)$, as in SK-Gd [2,3], for example. The large cross section of thermal neutron-capture on $\mathrm{Gd}(\mathrm{i} . \mathrm{e}$. $\mathrm{Gd}(\mathrm{n}, \gamma))$ is due to the contributions of two of its isotopes, ${ }^{155} \mathrm{Gd}(254000 \mathrm{~b})$ and ${ }^{157} \mathrm{Gd}$ (49000 b).

$$
\begin{aligned}
& n+{ }^{155} G d \rightarrow{ }^{156} G d^{*} \rightarrow{ }^{156} G d+\gamma \text { rays }(8.5 \mathrm{MeV} \text { total }) \\
& n+{ }^{157} G d \rightarrow{ }^{158} G d^{*} \rightarrow{ }^{158} G d+\gamma \text { rays }(7.9 \mathrm{MeV} \text { total })
\end{aligned}
$$

The emitted $\gamma$-spectrum and its corresponding Monte Carlo (MC) modelling for ${ }^{157} \mathrm{Gd}$ has already been discussed in [1]. However, the neutrino experiments use natural $\mathrm{Gd}\left({ }^{\mathrm{nat}} \mathrm{Gd}\right)$, which comprise of $14.80 \%$ of ${ }^{155} \mathrm{Gd}$, and $15.65 \%$ of ${ }^{157} \mathrm{Gd}$. We present the spectra from ${ }^{155} \mathrm{Gd}$, and ${ }^{\text {nat }} \mathrm{Gd}$, modify our "ANNRI-Gd" model with the contributions from ${ }^{155} \mathrm{Gd}$ and present our final MC for ${ }^{n a t} \operatorname{Gd}(\mathrm{n}, \gamma)$.

\section{Experiment and data analysis}

We measured the emitted $\gamma$-spectra from $\operatorname{Gd}(\mathrm{n}, \gamma)$ with the Accurate Neutron-Nucleus Reaction Measurement Instrument (ANNRI spectrometer) [4, 6, 7, 5, 8] in BL04 of JPARC-MLF facility. ANNRI is located $21.5 \mathrm{~m}$ away from the neutron beam source, and comprise of two clusters, each with 7 germanium (Ge) crystals, 8 co-axial detectors and Bimuth Germanium Oxide (BGO) anti-coincidence shields. We report the data, which was taken in March 2013 and Dec. 2014. The two main target samples were $\mathrm{Gd}_{2} \mathrm{O}_{3}$ enriched with $91.85 \%{ }^{155} \mathrm{Gd}$, and a metal film of ${ }^{\text {nat }} \mathrm{Gd}$ with $99.9 \%$ purity. We analysed our data for neutron energies 4-100 meV. The data was grouped into samples according to the clustering algorithm described in [1]. We calibrated the detector using the sources ${ }^{60} \mathrm{Co},{ }^{137} \mathrm{Cs},{ }^{152} \mathrm{Eu},{ }^{35} \mathrm{Cl}(\mathrm{n}, \gamma)$. We show the spectra of the most dominant sample (detected multiplicity $(\mathrm{M})=1$ and number of crystal hit $(\mathrm{H})=1$ ) after subtracting the corresponding background in figure 1. The background was measured with the empty target holder in the n-beam. The spectrum for ${ }^{157} \mathrm{Gd}$ is also shown for reference or reminder.

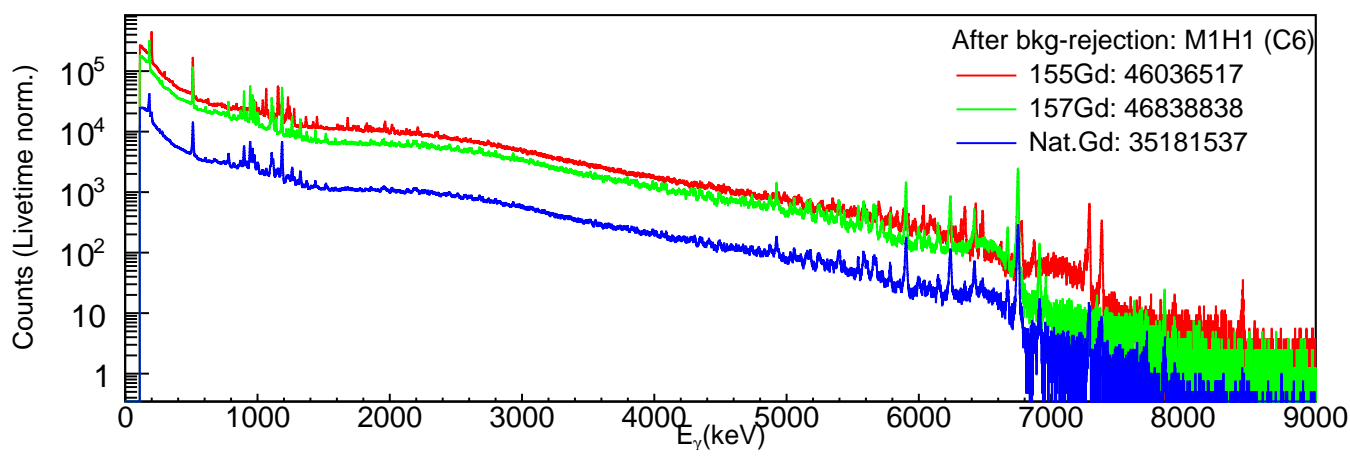

Figure 1: Single energy hit spectrum or M1H1 Spectra with Gadolinium samples, after subtracting the background, from one of the crystals. The numbers are the data statistics. 


\section{Modifying the ANNRI-Gd MC Model}

The MC model for ${ }^{157} \mathrm{Gd}$ is already described in [1]. We now develop the same for ${ }^{155} \mathrm{Gd}$. We identified and measured the photo peak intensities of 12 discrete $\gamma$ rays for ${ }^{155} \mathrm{Gd}(\mathrm{n}, \gamma)$ above $5 \mathrm{MeV}$ as in table 1, and found them in agreement with the values from ENSDF [9]. The discrete peaks contribute only $2.78 \pm 0.02 \%$ of the spectrum . The rest but dominant contribution of $97.22 \pm 0.02 \%$ comes from the continuum region of the energy levels in ${ }^{156} \mathrm{Gd}^{*}$.

The modelling of the continuum part uses the Standard Lorentzian PSF model, i.e., it basically employs the Fermi's Golden rule. The probability $(\mathrm{P})$ of transitioning from level $\mathrm{E}_{a}$ to level $\mathrm{E}_{b}$ emitting $\gamma$-ray $\left(\mathrm{E}_{\gamma}\right)$ given by "Transmission coefficient T" and "No. of levels $\rho\left(\mathrm{E}_{b}\right) \Delta \mathrm{E}_{b}$ "

$$
P_{a}\left(E_{a}, E_{b}\right) \Delta E_{b}=\frac{T\left(E_{a}, E_{b}\right)\left[\rho\left(E_{b}\right) \Delta E_{b}\right]}{\int_{0}^{E_{a}} T\left(E_{a}, E_{b}\right) \rho\left(E_{b}\right) d E_{b}}
$$

where $T\left(E_{a}, E_{b}\right)$ refers the Photon strength function (PSF) depending on cross sec. $\left(\sigma_{i}\right)$ and width $\left(\Gamma_{i}\right)$ of energy level $\left(E_{i}\right)$

$$
T\left(E_{\gamma}\right)=2 E_{\gamma}^{3} \frac{1}{3 \pi(\hbar c)^{2}} \sum_{i=1}^{2} \frac{\sigma_{i} E_{\gamma} \Gamma_{i}^{2}}{\left(E_{\gamma}^{2}-E_{i}^{2}\right)^{2}+E_{\gamma}^{2} \Gamma_{i}^{2}}
$$

with $\mathrm{E}_{i}=(11.2,15.2) \mathrm{MeV}, \sigma_{i}=(180,242) \mathrm{mb}$ and Width $\left(\Gamma_{i}\right)=(2.6,3.6) \mathrm{MeV}[10,11]$.

Spectral components of the discrete part are added and tuned with that of ${ }^{155} \mathrm{Gd}$ data. The continuum and the discrete component generated by our model shown separately here for ${ }^{155} \mathrm{Gd}$, along with the data in figure 2-top. They are added in corresponding proportion to generate the final ${ }^{155} \mathrm{Gd}(\mathrm{n}, \gamma)$ spectrum in figure 2-bottom. The data spectrum matches well with our MC spectrum.

\begin{tabular}{|c|c|c|}
\hline & 1 st $\gamma(\mathrm{MeV})$ & Intensity (\%) \\
\hline 1 & 8.448 & $0.018 \pm 0.002$ \\
\hline 2 & 7.382 & $0.233 \pm 0.018$ \\
\hline 3 & 7.288 & $0.453 \pm 0.026$ \\
\hline 4 & 6.474 & $0.352 \pm 0.007$ \\
\hline 5 & 6.430 & $0.324 \pm 0.027$ \\
\hline 6 & 6.348 & $0.303 \pm 0.026$ \\
\hline 7 & 6.319 & $0.094 \pm 0.005$ \\
\hline 8 & 6.034 & $0.204 \pm 0.019$ \\
\hline 9 & 5.885 & $0.174 \pm 0.029$ \\
\hline 10 & 5.779 & $0.188 \pm 0.008$ \\
\hline 11 & 5.698 & $0.286 \pm 0.008$ \\
\hline 12 & 5.661 & $0.154 \pm 0.007$ \\
\hline
\end{tabular}
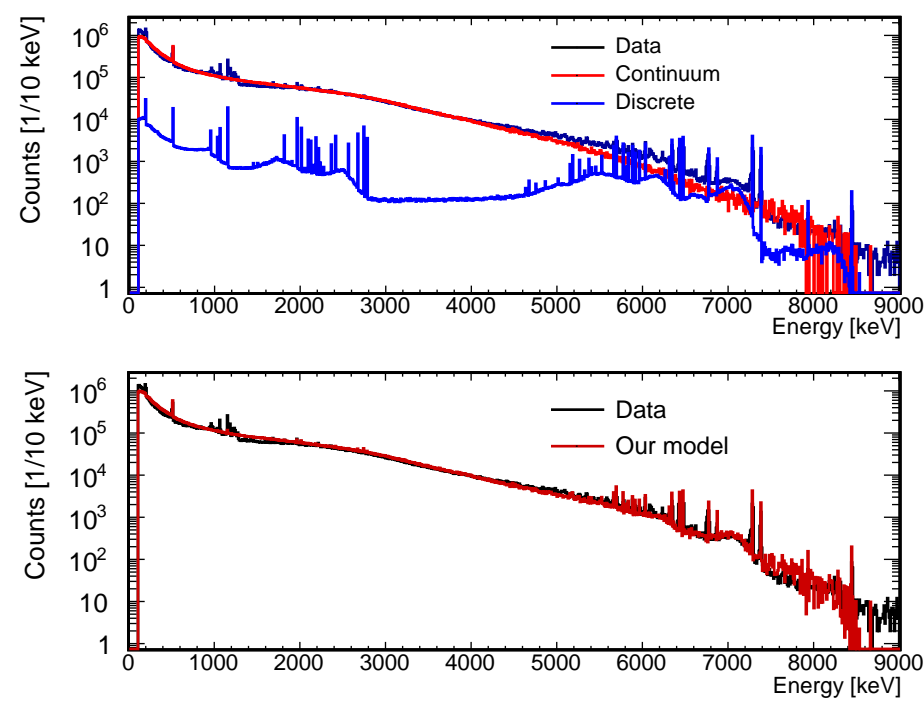

Table 1: The relative intensities of the 12 primary discrete peaks of ${ }^{155} \mathrm{Gd}(\mathrm{n}, \gamma)$.

Figure 2: Top: The discrete (blue) and continuum (red) component of our model for ${ }^{155} \mathrm{Gd}(\mathrm{n}, \gamma)$ shown separately, along with the data (black). Bottom: Single energy hit spectrum from data (black) and our MC model (red) for ${ }^{155} \mathrm{Gd}(\mathrm{n}, \gamma)$. 


\section{Results and Summary}

We finally generate the spectrum for ${ }^{\text {nat }} \mathrm{Gd}(\mathrm{n}, \gamma)$, by adding the same generated by our model for ${ }^{155} \mathrm{Gd}(\mathrm{n}, \gamma)$ and ${ }^{157} \mathrm{Gd}(\mathrm{n}, \gamma)$ in the required ratio of their relative cross-section and abundance, as shown in figure 3-left. The corresponding spectra generated by GLG4sim [12] and the GEANT4photon evaporation model $[13,14]$ are also shown. The spectrum generated by our "ANNRI-Gd" MC-model agrees better than most other available MC generators for $\mathrm{Gd}(\mathrm{n}, \gamma)$. We also show the ratio of data/MC in bins of $200 \mathrm{keV}$ for ${ }^{155} \mathrm{Gd},{ }^{157} \mathrm{Gd}$, and ${ }^{\text {nat }} \mathrm{Gd}$ in figure 3-right, as an approximate representation of the goodness of our model.
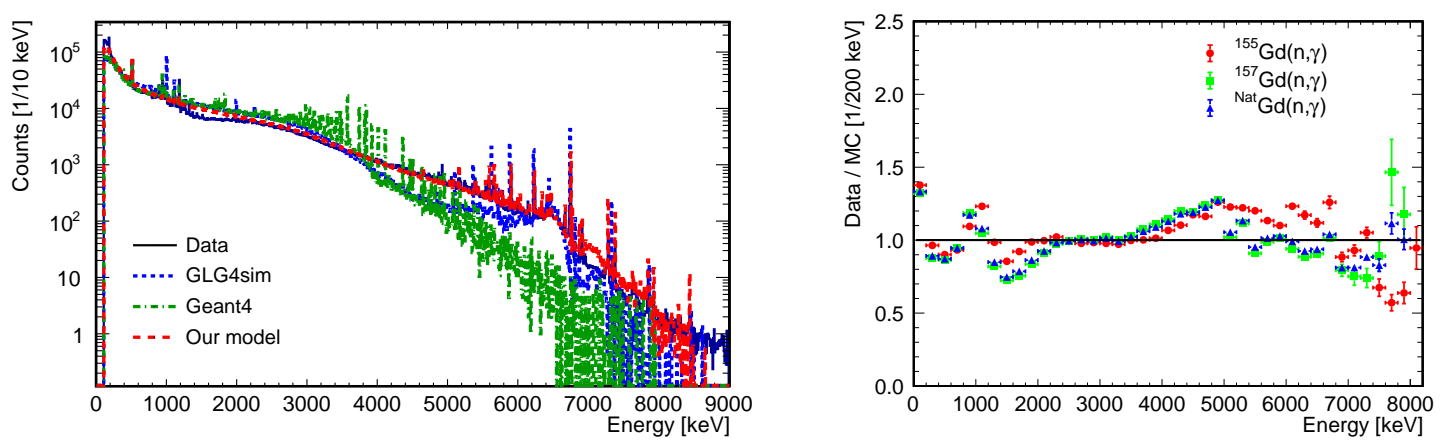

Figure 3: Left: Single energy hit spectra from data (black) and our MC model (red) for ${ }^{\text {nat }} \mathrm{Gd}(\mathrm{n}, \gamma)$. The corresponding spectra from GLG4sim (blue) and GEANT4 (green) are also shown. Right: Data/MC (our model) in bins of $200 \mathrm{keV}$ for ${ }^{155} \mathrm{Gd}(\mathrm{n}, \gamma)$ (red), ${ }^{157} \mathrm{Gd}(\mathrm{n}, \gamma)$ (green) and ${ }^{\text {nat }} \mathrm{Gd}(\mathrm{n}, \gamma)$ (blue).

\section{References}

[1] K. Hagiwara et al. arXiv:1809.02664 [nucl.ex]. .

[2] Sekiya, Hiroyuki for the [Super-Kamiokande Collaboration]. PoS, ICHEP2016:982, 2016.

[3] Watanabe, H. et al. [Super-Kamiokande Collaboration]. Astropart. Phys., 31(4):320-328, 2009.

[4] M. Igashira, Y. Kiyanagi, and M. Oshima. Nucl. Instrum. Meth., A600(1):332-334, 2009.

[5] Kimura, A. et al. J. Nucl. Sci. Technol., 49(7):708-724, 2012.

[6] Kin, T. et al. J. Korean Phys. Soc., 59:1769-1772, 2011.

[7] Kino, K. et al. Nucl. Instrum. Meth., A626:58-66, 2011.

[8] Kino, K. et al. Nucl. Instrum. Meth., A736:66-74, 2014.

[9] N. Nica. Nuclear Data Sheets for A=158. Nucl. Data Sheets, 141:1-326, 2017.

[10] J. Kopecky, M. Uhl, and R. E. Chrien. Phys. Rev. C, 47:312-322, Jan 1993.

[11] Japan Atomic Energy Agency. Japanese Evaluated Nuclear Data Library 4.0 (JENDL-4.0) Homepage. http://www.ndc.jaea.go.jp/jendl/j40/j40.html.

[12] Smith, G. et al. GLG4sim, Generic liquid-scintillator anti-neutrino detector Geant4 simulation. http://neutrino.phys.ksu.edu/ GLG4sim/.

[13] Agostinelli, S. et al. [Geant4 Collaboration]. Nucl. Instrum. Meth., A506:250-303, 2003.

[14] J. Allison et al. Nucl. Instrum. Meth., A835:186-225, 2016. 